\title{
Performance comparison of guidance strategies to accomplish RTAs during a CDO
}

\author{
Ramon Dalmau and Xavier Prats \\ Department of Physics - Aeronautics Division \\ Technical University of Catalonia - BarcelonaTECH \\ Castelldefels, Spain
}

\author{
Ronald Verhoeven, Frank Bussink and Bart Heesbeen \\ Department of Cockpit and Flight Operations \\ Netherlands Aerospace Centre (NLR) \\ Amsterdam, The Netherlands
}

\begin{abstract}
Continuous Descent Operations with Controlled Times of Arrival (CTA) at one or several metering fixes could enable environmentally friendly procedures without compromising capacity. This type of flight operation requires advanced on-board systems not only able to compute a plan satisfying Required Times of Arrival (RTAs), but also to safely and efficiently guide the aircraft during the execution of the descent such that RTAs are accurately accomplished. The primary aim of this paper is to compare the performance (in terms of environmental impact mitigation and ability to fulfill operational constraints) of four guidance strategies: tactical, strategic, hybrid and Model Predictive Control (MPC). A high fidelity flight simulator has been configured, and several descents to Barcelona-El Prat airport (Spain) have been simulated in presence of weather forecast and aircraft performance modeling errors. Results show that MPC is the most robust in terms of energy and time deviation, providing at the same time excellent environmental impact mitigation.
\end{abstract}

\section{INTRODUCTION}

Continuous Descent Operations (CDO) have been subject of extensive research in the last decades. Even if proving successful in reducing the environmental impact [1], [2], such operations suffer from a well known drawback: the loss of predictability from the Air Traffic Control (ATC) point of view, in terms of overfly-times at certain fixes along the lateral route.

Consequently, existing CDO implementations require ATC to introduce additional sequencing buffers to ensure safe separation among aircraft, thus reducing airport capacity. For these reasons, in some busy airports CDOs are only operational in off-peak hours, when the traffic demand is low [3].

A potential approach to enable CDO in dense traffic scenarios consists on assigning CTAs to each aircraft at some strategic fixes for separation, sequencing and merging tasks. With this type of flight operations, ATC will assign a CTA to each aircraft before reaching the Top Of Descent (TOD). This assigned control time will be entered as a Required Time of Arrival (RTA) by the Flight Management System (FMS). Then, the on-board trajectory predictor will compute the optimal plan in terms of e.g. fuel while satisfying the RTA [4].

Because of modeling errors and uncertainties encountered during the execution of the descent, aircraft may deviate from the planned altitude and/or speed profile, and may also be delayed or advanced with respect to the planned time schedule. In order to safely and accurately satisfy the RTA, corrective actions must be taken by the guidance system.
Various guidance strategies can be used to satisfy RTA, depending on how the elevator and throttle act together to nullify deviations. For instance, the speed could be controlled with throttle commands to tactically nullify time deviations (speed-on-throttle), using the elevator to follow the planned path (path-on-elevator) [5]. This continuous control on thrust has a negative effect on noise nuisance and fuel usage [6].

A more efficient guidance strategy in terms of environmental impact consists on actively controlling the speed with the elevator to tactically nullify time deviations (speed-on-elevator), using near-idle thrust variations to recover the path whenever the vertical deviation exceeds a pre-defined threshold [7].

Other guidance strategies suitable to accomplish RTAs during a CDO have been proposed as a part of the Time and Energy Managed Operations (TEMO) concept [8], [9]. These strategies have in common to manage the energy of the aircraft (altitude and speed) such that time constraints are satisfied.

For the tactical TEMO variant, the elevator is in charge of nullifying time errors by controlling the speed, while throttle and speed-brakes continuously act to correct energy deviations.

For the strategic TEMO variant [8], the plan is executed using speed-on-elevator and thrust-on-throttle, allowing certain energy and time deviations. Whenever these deviations exceed a predefined threshold, a new plan that takes corrective actions to satisfy the RTA minimizing fuel consumption and speedbrakes usage is generated starting from the current position.

In previous work [10], this technique was compared with the behavior of a typical flight management system dormant to time errors, showing superior performances in terms of fuel consumption and RTA adherence in presence of wind errors.

A combination of the strategic and tactical TEMO variants leads to the hybrid guidance [9]. This strategy consists of executing the plan using thrust-on-throttle and a tactical controller to nullify time errors with the elevator. Eventual energy deviations are corrected by means of strategic re-plannings.

Finally, using the Model Predictive Control (MPC) [11] variant the plan is regularly optimized without waiting for deviations to exceed a certain threshold.

To the best of our knowledge, a comprehensive comparison of guidance strategies to accomplish RTAs during a CDO has not been performed before. This paper compares the performance of the tactical, strategic, hybrid and MPC strategies 
in terms of environmental impact and the ability to satisfy operational constraints (including applicable RTAs).

In order to accomplish that, several descents subject to weather forecast and aircraft performance model errors have been simulated using a high fidelity flight simulator, configuring its guidance system with these strategies.

\section{BACKGROUND}

Flight management systems suitable for time-constrained CDOs require a trajectory predictor capable of generating, in real-time, an optimal plan satisfying eventual RTAs and other applicable constraint; either when the RTAs are entered in cruise or when a plan update is required during the descent.

Section II-A describes the optimization method used by the trajectory predictor implemented in the flight simulator. The working principles of the four guidance strategies considered herein are presented in Section II-B.

\section{A. Optimal trajectory planning}

The trajectory predictor formulates the optimization of the trajectory as an optimal control problem, using a three degrees of freedom point-mass model to describe aircraft dynamics.

Section II-A1 presents the generic formulation of an optimal control problem, while Section II-A2 describes the equations of motion that mathematically represent the aircraft dynamics.

1) Optimal control formulation: The goal of an optimal control problem is to find the time history of the control vector $\boldsymbol{u}(t)$ and the time-independent parameters $\boldsymbol{p}$ of a system with a state vector $\boldsymbol{x}(t)$ such that a cost function $J$, defined over an either free or fixed time interval $\left[t_{0}, t_{f}\right]$, is minimized [12].

In order to guarantee a feasible and operational trajectory, as a result of the optimization process, several constraints must be considered. In particular, the dynamics of the system $\boldsymbol{f}$ expressed by non-linear vector functions:

$$
\frac{\mathrm{d} \boldsymbol{x}}{\mathrm{d} t}=\dot{\boldsymbol{x}}(t)=\boldsymbol{f}(\boldsymbol{x}(t), \boldsymbol{u}(t), \boldsymbol{p}, t)
$$

In addition, $\boldsymbol{x}$ is typically fixed to some initial conditions:

$$
\boldsymbol{x}\left(t_{0}\right)=\boldsymbol{x}_{0}
$$

and the solution might also satisfy some terminal constraints:

$$
\boldsymbol{\psi}\left(\boldsymbol{x}\left(t_{f}\right), t_{f}\right)=0
$$

algebraic path constraints:

$$
\boldsymbol{g}(\boldsymbol{x}(t), \boldsymbol{u}(t), \boldsymbol{p}, t) \leq 0
$$

and box constraints (also known as bounds) on the state, control and parameter variables:

$$
\begin{aligned}
\boldsymbol{x}_{L}(t) & \leq \boldsymbol{x}(t) \leq \boldsymbol{x}_{U}(t) \\
\boldsymbol{u}_{L}(t) & \leq \boldsymbol{u}(t) \leq \boldsymbol{u}_{U}(t) \\
\boldsymbol{p}_{L} & \leq \boldsymbol{p} \leq \boldsymbol{p}_{U}
\end{aligned}
$$

In the previous notation, $(\cdot)_{L}$ and $(\cdot)_{U}$ are respectively the lower and upper bounds of these box constraints.
For those problems defined over more than one phase, the dynamics of the system, the terminal, path and box constraints might be different in each phase. However, it might be desirable to link the state variables across two consecutive phases. This is accomplished by enforcing additional link constraints.

There are several ways to solve the optimal control problem (1)-(5). In this paper direct collocation methods have been used. Such direct methods transform the original continuous (and thus infinite) optimal control problem into a (discrete and finite) Non-Linear Programming (NLP) problem[13], [14].

The time histories of control and state variables are discretized at a set of collocation points, being the differential equations (1) approximated by some continuous function (such as polynomials) over each collocation step. The values of these discretized variables, along with $\boldsymbol{p}$ and/or $t_{f}$, become the unknowns of the new finite variable problem, which can be formulated as a NLP problem and solved by NLP solvers.

2) Aircraft dynamics model: The trajectory predictor expresses the dynamics of the aircraft with the following three degrees of freedom point-mass model:

$$
\begin{aligned}
\frac{\mathrm{d} v}{\mathrm{~d} t} & =\dot{v}=\frac{T(\pi)-D(\beta)}{m}-g \sin \gamma \\
\frac{\mathrm{d} \gamma}{\mathrm{d} t} & =\dot{\gamma}=\frac{g}{v}\left(n_{z}-\cos \gamma\right) \\
\frac{\mathrm{d} h}{\mathrm{~d} t} & =\dot{h}=v \sin \gamma \\
\frac{\mathrm{d} s}{\mathrm{~d} t} & =\dot{s}=\sqrt{v^{2} \cos ^{2} \gamma-W_{x}^{2}}+W_{s} \\
\frac{\mathrm{d} m}{\mathrm{~d} t} & =\dot{m}=-f
\end{aligned}
$$

where the state vector $\boldsymbol{x}=[v, \gamma, h, s, m]$ is composed by the true airspeed (TAS), the aerodynamic flight path angle, the geometric altitude, the distance to go and the mass of the aircraft; the control vector $\boldsymbol{u}=\left[\pi, \beta, n_{z}\right]$ is composed, respectively, by the throttle setting, the speed-brakes deflection and the load factor $\left(n_{z}=\frac{L}{m g}\right) ; T$ is the total thrust; $D$ is the aerodynamic drag; $L$ is the aerodynamic lift; $f$ is the total fuel flow; $W_{x}$ and $W_{s}$ are, respectively, the cross and along path wind components; and $g$ is the local gravity acceleration.

Note that the effects of the bank angle, the vertical wind and the wind speed derivatives have been neglected in Eq. (6), aiming to reduce the complexity of the mathematical model and, consequently, the computational burden of NLP problem.

Regarding the atmosphere, the International Standard Atmosphere (ISA) model is considered, which defines the density, pressure and temperature magnitudes as a function of $h$.

\section{B. Time and energy managed guidance strategies}

The total energy of the aircraft $\left(E_{t}\right)$ is composed by the sum of its kinetic $\left(E_{k}\right)$ and potential $\left(E_{p}\right)$ energy:

$$
E_{t}=E_{k}+E_{p}=\frac{1}{2} m v^{2}+m g h
$$

By differentiating Eq. (7) and combining it with Eqs. (6a) and $(6 c)$, the energy rate of the aircraft can be derived as: 


$$
\dot{E}_{t}=v(T-D)
$$

According to Eq. (8), the total energy of an aircraft can be increased by applying thrust, and decreased by increasing drag. In addition, the law of conservation of energy states that potential energy can be exchanged for kinetic energy and vice versa through energy modulation. It is well known that throttle and speed-brakes are the most effective means to increase and decrease the total energy of the aircraft, whereas elevator control provides an effective mean to modulate energy.

The core principle of the TEMO concept is that the energy of the aircraft is managed in such a way that the RTAs are fulfilled: speed-on-elevator is used to exchange potential (altitude) and kinetic energy (speed) at an appropriate rate, while throttle and speed-brake are only applied when energy needs to be added or removed from/to the system, respectively.

A TEMO descent can be executed using several guidance variants, depending on how time and energy errors are treated:

1) Tactical guidance: The deviations from the planned time at the current position are continuously nullified by using speed-on-elevator, while the throttle and the speed-brakes act together to maintain the planned energy level. Using this strategy, the aircraft is continuously following the initially computed plan with minimum energy and time deviations, thus there is no need to update it during the course of the descent.

This logic typically has a negative impact on the environment because it is more likely to use thrust and speed-brakes.

2) Strategic re-planning: The calibrated airspeed plan (CAS) is executed using speed-on-elevator and thrust-onthrottle, allowing time and energy deviations as long as they do not exceed a predefined threshold. Otherwise, the trajectory predictor optimizes again the trajectory, enforcing the initial conditions to the current state and time. This results in a new CAS plan that minimizes fuel consumption and speed-brakes use while satisfying all the constraints (including RTAs).

An advantage of the strategic guidance is that the entire remaining trajectory is taken into account when correcting deviations. In addition, being dormant with respect to time and energy deviations could lead to positive effects if the errors caused by different sources of uncertainty are counteractive.

3) Hybrid guidance: This strategy is obtained as a result of combining the tactical and the strategic guidances.

A tactical controller nullifies sustained time errors using speed-on-elevator, and the throttle executes the thrust plan. Energy deviations are corrected by means of strategic replanning whenever the pre-defined threshold is exceeded. As a result, the aircraft is no longer affected by time errors, at the expense of being more prone to energy deviations.

4) Model predictive control (MPC): This guidance strategy is based on the repeated solution of the open-loop optimal control problem described in Section II-A1. Based on the current state of the aircraft, the optimal control problem is solved over a finite time horizon in the future, and the resulting CAS plan is executed using speed-on-elevator and thrust-onthrottle only until the next re-plan instant. Then, the process is repeated moving the time horizon forward.
Depending on how often the plan is updated, different variants of MPC exist. If the optimal control problem is solved at all time instants, it is referred as instantaneous MPC. If it is solved only at disjoint instants and the resulting plan is executed in between, the strategy is called sampled-data MPC.

Another way of classifying MPC is according to the size of the time horizon. Considering this criteria, MPC can be divided into two major classes: In a receding horizon MPC (RHMPC) [15], the size of the time horizon is constant and relatively small; in a shrinking-horizon MPC (SHMPC), the entire remaining trajectory is optimized, thus the time horizon length decreases while approaching the terminal state.

For the specific problem of an aircraft (a system with relatively slow dynamics) that needs to achieve a fix in space and time, sampled-data SHMPC is considered the best choice.

The execution time and convergence of a NLP problem strongly depend on its initial guess. For the SHMPC, the part of the old solution that corresponds to the new horizon could be used to initialize the variables of the subsequent optimization problem, significantly speeding-up the computational time. This approach is commonly known as warm start [16].

\section{SET UP OF THE EXPERIMENT}

The primary scope of this experiment is to compare the performance of the guidance strategies presented in Section II-B to provide accurate, safe and efficient aircraft guidance towards a single time-constrained metering fix during a CDO.

The comparison has been performed for a realistic scenario, and the effects of weather forecast and performance modeling errors have been investigated through a batch simulation.

Section III-A presents the scenario considered in this paper. Section III-B describes the constraints and cost function that set up the trajectory optimization problem. Section II-B gives a rundown of the parameters chosen to configure each guidance strategy. Section III-D lists the selected weather forecast and aircraft performance modeling errors.

\section{A. Scenario}

The experiment has been performed for Barcelona-El Prat airport (Spain) using four common Standard Terminal Arrival Routes (STARs) at runway $25 \mathrm{~L}$ in west configuration, which were designed to perform CDOs during nightly hours. These STARs end at four different initial approach fixes and merge into a single intermediate fix (SOTIL), which has been selected as the time-constrained metering fix for the experiment.

An Airbus A320-alike model has been used for the experiment. It should be noted that even if both flight simulator and trajectory predictor draw upon the same aircraft type, the dynamics and performance model used by these components are sightly different: the flight simulator uses as a high fidelity six degrees of freedom model to represent the aircraft dynamics, and look-up tables for the aircraft performance; the trajectory predictor represents the aircraft as a three degrees of freedom point-mass model, and approximates the performance tables with polynomial functions, aiming to achieve the continuity and differentiability required by state-of-the-art NLP solvers. 


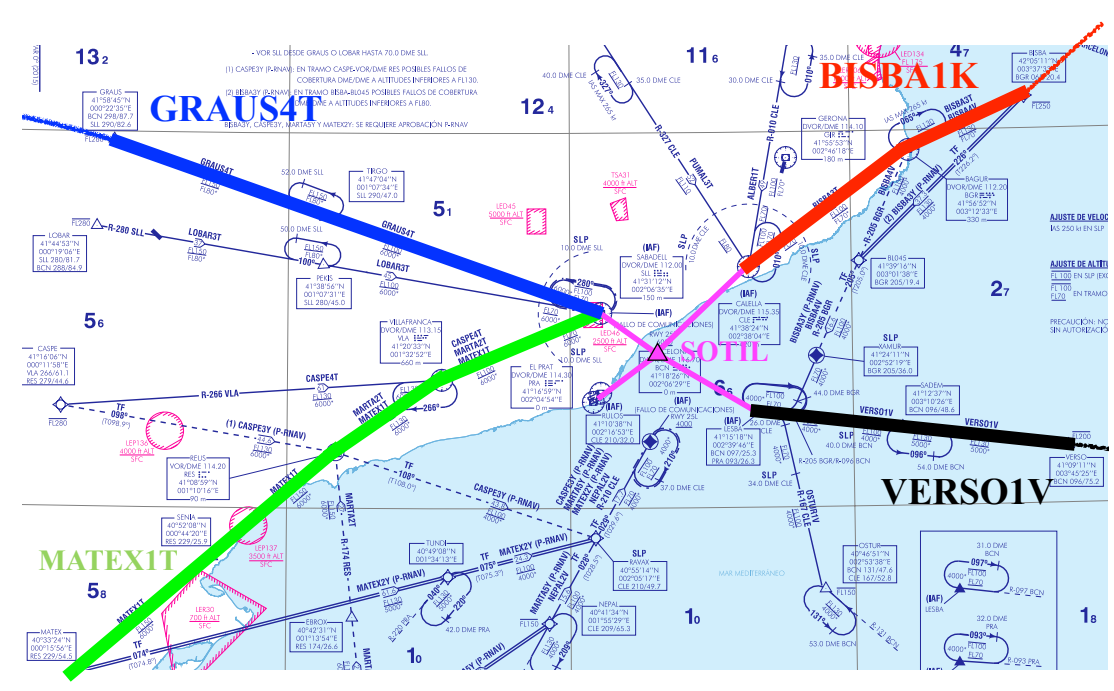

Fig. 1: Routes chosen for the experiment (Source: Spanish AIP)

All simulations start at a distance to go of $140 \mathrm{NM}$, cruising at FL360 and Mach 0.78, and with a mass corresponding to $90 \%$ of the maximum landing mass. Immediately, the CTA is received from an hypothetical ATC, and a re-plan is triggered to generate an optimal plan satisfying the enetered RTA.

Three different CTAs have been considered in this paper. These CTAs enforce to arrive at SOTIL in 21, 22 and 23 minutes from the start of the simulation, respectively.

Few nautical miles before SOTIL the pseudo-pilot pressed the approach button, arming in this way the localizer and instrumental landing system (ILS) glide path modes. All the simulations end when SOTIL is reached and all the metrics used for the comparison are computed at this fix.

\section{B. Flight profile}

The trajectory predictor generates the optimal descent plan subject to RTAs by solving a constrained non-linear optimal control problem, as described in Section II-A. Aiming at reducing the environmental impact, the objective function is to minimize fuel consumption and speed-brakes use:

$$
J=\int_{t_{0}}^{t_{f}} F F(t)+\beta(t) \mathrm{d} t
$$

Constraints on the aircraft dynamics are particularized by the point-mass model described by Eq. (6), and simple box constraints on the controls are specified as follows:

$$
0 \leq \pi \leq 1 ; \quad \beta \in\{0,1\} ; \quad 1.15 \leq n_{z} \leq 0.85
$$

where $\pi=0$ and $\pi=1$ correspond to idle and maximum thrust, respectively; and $\beta$ is a binary variable that can only take two levels: either retracted (0) or deployed (1). Bounds on $n_{z}$ have been chosen to provide good passengers comfort [17].
Generic path constraints as a function of the state variables ensure that the calibrated airspeed $\left(v_{C A S}\right)$ and Mach number $(M)$ remain within operational limits all along the descent:

$$
\mathrm{VMO} \leq v_{C A S} ; \quad \mathrm{MMO} \leq M
$$

where VMO and MMO are, respectively, the maximum operational $v_{C A S}$ and $M$. The descent is split in four different phases, where additional phase-dependent box, path and/or terminal constraints may apply.

The first phase, performed at constant Mach and pressure altitude $\left(h_{p}\right)$, is followed by a cruise speed adjustment phase that ends at the TOD, where the aircraft starts the descent towards SOTIL. Before reaching this fix, the aircraft configures with flaps at the green dot speed (GD) ${ }^{1}$. Then,

The last phase of the profile ends with the aircraft at SOTIL, at $\mathrm{S}$ speed $^{2}$ and at 3,000 $\mathrm{ft}$, being ready to transition to the next flaps setting, intercept the ILS glide path and land. Obviously, the RTA is modeled by fixing the final time of this last phase.

It should be noted that the optimal trajectory in terms of fuel consumption tends to fly at very high altitudes and low speeds during most of the descent, releasing the leftover energy at low altitudes by increasing the airspeed and, consequently, the drag. Finally, a level-off at idle thrust is performed to achieve a fast deceleration and satisfy the terminal condition.

This solution is neither operationally realistic nor optimal in terms of noise. In order to face this issue and achieve a smooth deceleration, the lower and upper limits on the flight path angle after the TOD have been set to $-3^{\circ}$ and $-1^{\circ}$, respectively. This restriction will penalize all the guidance strategies in the same way, not impacting on the comparative results.

\footnotetext{
${ }^{1}$ Green dot speed is the minimum operating speed in managed mode and clean configuration, being approximately the best lift-to-drag ratio speed.

${ }^{2} \mathrm{~S}$ speed is the target speed when the aircraft is in configuration 1.
} 


\section{Configuration of the guidance strategies}

The tactical guidance logics have been configured to continuously adjust the commanded CAS such that time deviations are nullified using speed-on-elevator. Regarding the specific energy (defined as the total energy divided by the weight of the aircraft), a maximum allowed deviation of $50 \mathrm{ft}$ has been set. In the case of being above this threshold, speed-brakes are deployed; in the case of falling below, the throttle is used to recover the planned energy level by commanding small amounts of thrust. Under no circumstances the trajectory will be updated during the execution of the descent.

The specific energy deviation bounds for the strategic and hybrid guidances have been set to $500 \mathrm{ft}$ in the cruise phase until the TOD and $100 \mathrm{ft}$ at the metering fix, while time deviation bounds for the strategic guidance have been set to 10 and 3 seconds, respectively. Between these two points, specific energy and time deviation bounds were linearly interpolated.

Finally, the SHMPC has been configured to update the plan every 30 seconds, regardless of the present energy and time deviations. This strategy is activated at the beginning of the descent, aiming to avoid a continuous shift of the TOD.

\section{Weather forecast and performance modeling errors}

The trajectory predictor has been configured to compute the descent plan assuming ISA conditions, calm winds and nominal aircraft performance models. Then, wind and/or nonstandard atmospheric conditions are incorporated during the execution of the descent to mimic mismatches between the prediction and the reality. Similarly, the aircraft performance models of the flight simulator have been modified by adding variations with respect to those used by the trajectory predictor.

1) Weather forecast errors: The accuracy of the plan computed by the trajectory predictor, and especially the computation of the estimated time of arrival over the metering fix, critically depends on the quality of the weather forecast.

In this paper, positive and negative temperature errors of 5 and $10^{\circ} \mathrm{C}$ and wind speed errors of 5 and $10 \mathrm{kt}$ have been considered, while pressure errors are left for future work.

It is important to remember that the trajectory predictor computes the plan considering ISA and calm winds. As result the temperature errors are translated to ISA deviations during the execution of the simulation. Similarly, wind speed errors are accomplished by simply adding winds that were not considered during the planning process.

The two wind speeds chosen for this experiment have been combined with the four compass wind directions, leading to a total of eight simulations with wind errors for each combination of guidance strategy, route and CTA.

Regarding the temperature, a single STAR have been considered because results are independent of the route, provided that the ISA deviation is not a function of the distance to go.

2) Aircraft performance models errors: Inaccuracies in the aircraft performance models are also a non-depreciable source of error when predicting trajectories. These models are composed by a set of parameterized functions or look-up tables that express aircraft characteristics (e.g. drag coefficient) as a function of certain variables (e.g. Lift coefficient and Mach).

In this paper the effects of errors in the drag coefficient and idle thrust models have been analyzed. These aircraft-related performance functions are expected to have the highest impact on the accuracy of the predicted CDO trajectory.

Overestimations and underestimations of 5\% in the magnitude of these two aircraft features have been considered in this experiment. Therefore, a total of four simulations with aircraft performance model errors have been executed for each combination of CTA and guidance strategy. As for the case with temperature errors, a single STAR has been considered for the analysis because results are independent of the route.

\section{RESUlts}

Data for the comparison of guidance strategies have been obtained as a result of a batch composed by 492 simulations.

Fuel consumption, time and energy deviation at SOTIL, number of speed-brakes deployments and area affected by the aircraft noise have been selected as metrics for the comparison.

Noise has been calculated using the model described in [18].

Section IV-A illustrates the working principle of the guidance strategies presented in Section II-B by means of a practical example of simulation. Section IV-B compares the performance of the guidance strategies subject to weather forecast and aircraft performance modeling errors.

\section{A. Examples}

The simulation chosen for this illustrative example corresponds to that executed by following the BISBA1K STAR, subject to a RTA requiring to arrive in 23 minutes, with roughly $10 \mathrm{kt}$ of unexpected head wind, in ISA conditions and without aircraft performance modeling errors. Figure 2 shows, for each guidance strategy, the time and specific energy deviations ( $\Delta t$ and $\Delta E_{s}$, respectively) along with their thresholds (if any), as a function of the distance to go until the metering fix.

Figures 3 and 4 show the planned and executed states and controls for the different guidance strategies, respectively.

According to Fig. 2(a), in tactical guidance the time error caused by the unexpected head wind is continuously nullified by using speed-on-elevator, while energy deviations are corrected by either deploying speed-brakes or adding minimum amounts of thrust whenever these deviations exceed $50 \mathrm{ft}$.

Figure 3(a) illustrates that using this mechanism the initial plan is never updated. Figure 4(a) shows that the throttle acted twelve times to correct a negative energy deviation, while speed-brakes were deployed twice due to an excess of energy.

In strategic guidance the CAS plan is executed using speedon-elevator and thrust-on-throttle, and neither time nor energy deviations are corrected as long as they remain within the allowed thresholds. Otherwise, the trajectory is re-calculated.

Fig. 2(b) shows that five re-plans were triggered by a time error exceeding the upper threshold (i.e. the aircraft was late).

Whenever a re-plan is triggered, the current state and time are enforced as the initial conditions for the subsequent 


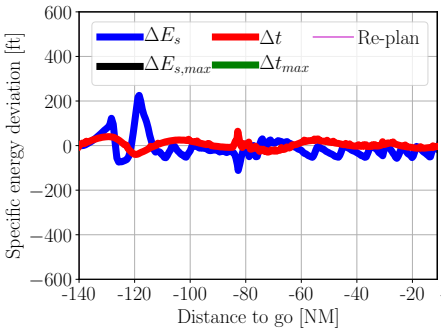

(a) Tactical

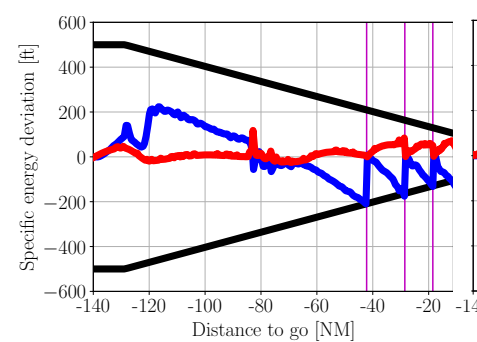

(c) Hybrid

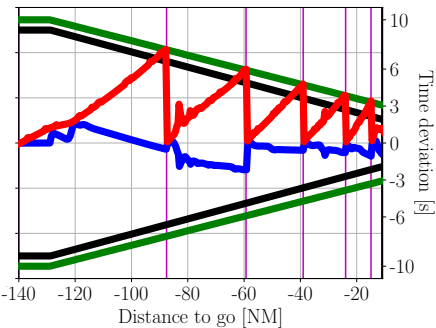

(b) Strategic

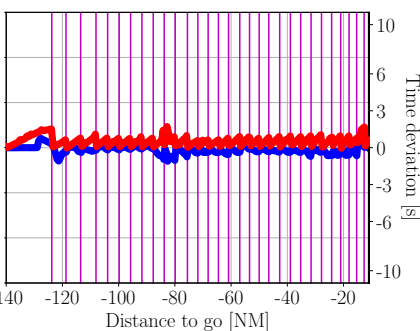

(d) SHMPC
Fig. 2: Specific energy and time deviations

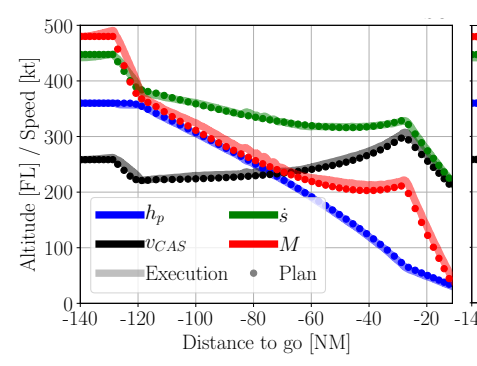

(a) Tactical

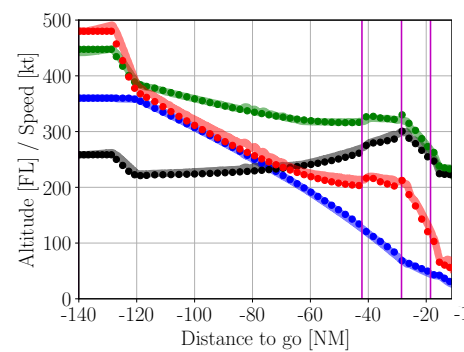

(c) Hybrid

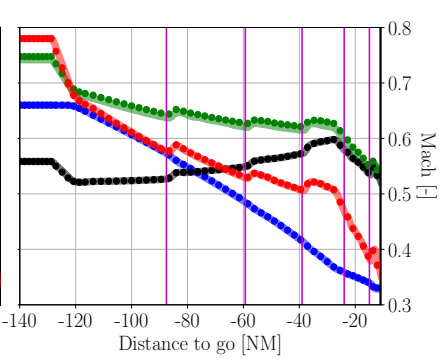

(b) Strategic

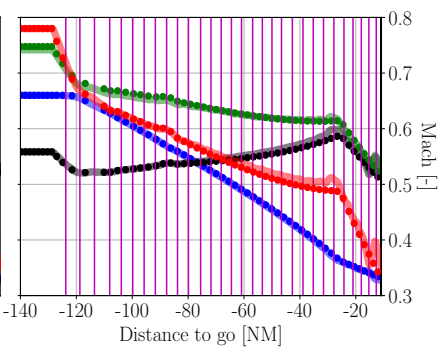

(d) SHMPC
Fig. 3: Planned and executed states

optimal control problem, thus both energy and time deviations are nullified at the moment the new plan becomes active.

According to Fig. 3(b), all re-plans claimed a noticeable faster speed profile if compared with what was initially planned, aiming to correct the accumulated time deviations.

Figure 4(b) shows that for none of the re-plans energy modulation with the elevator was sufficient to correct the accumulated time and energy deviations. All re-plans claimed to add a relatively small amount of energy to the system by means of thrust, aiming to increase the planned speed profile.

For the hybrid guidance a tactical controller nullifies time deviations with the elevator while following the throttle plan,

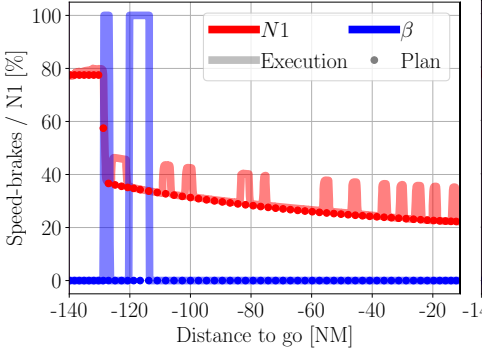

(a) Tactical

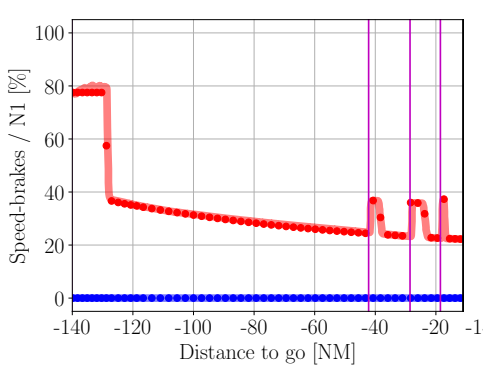

(c) Hybrid

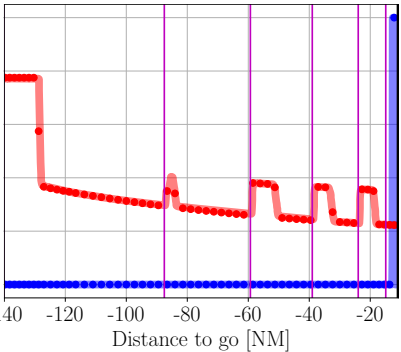

(b) Strategic

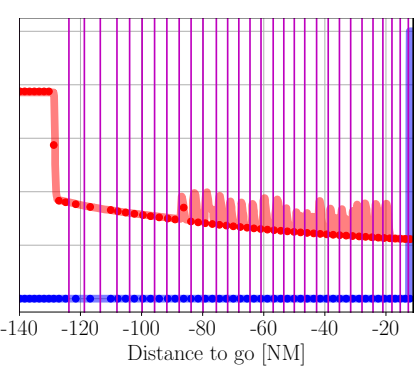

(d) SHMPC

Fig. 4: Planned and executed controls

and energy deviations are corrected by using strategic re-plans.

According to Fig. 3(c), the tactical controller systematically increases the CAS above that planned in order to nullify the time deviations caused by the unexpected wind. This higher speed leads to an increase of the drag and, consequently, to the energy loss rate. As a result, three re-plans were triggered by an excessive energy deviation reaching the lower threshold.

It should be noted that due to the dormant behavior of this strategy with respect to energy deviations as long as the allowed threshold is not overpassed, the metering fix is achieved almost $100 \mathrm{ft}$ below the planned specific energy level. This missing energy will need to be recovered afterwards by means of additional thrust, leading to more fuel consumption and noise nuisance at low altitudes close to populated areas.

For the SHMPC strategy, energy and time deviations are neither tactically nor strategically corrected during the course of the descent (see Fig. 2(d)). Instead, the trajectory is frequently updated by solving the open-loop optimal control problem described in Section II-A1 over the remaining descent.

In this particular example, the plan was updated 30 times, reaching SOTIL at the planned time and energy level.

According to Fig 4(d), energy modulation was sufficient to correct energy and time deviations until $90 \mathrm{NM}$ from the runway threshold. Thereafter, small (but optimal) amounts of energy were added to the system after each re-plan, aiming to correct energy deviations and adjust the speed plan.

If compared with the hybrid and strategic re-plans, SHMPC re-plans lead to less modifications of the active plan, thus requiring less throttle and speed-brakes use. The reason behind this fact is that time and energy deviations at the moment the re-plan is triggered are smaller, because they are continuously corrected rather than waiting them to reach a certain threshold. 


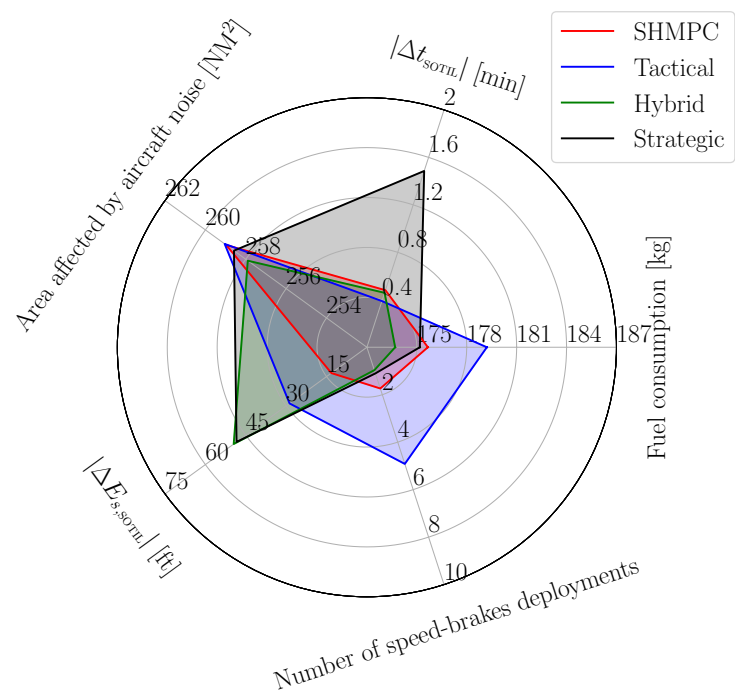

(a) Aggregated results

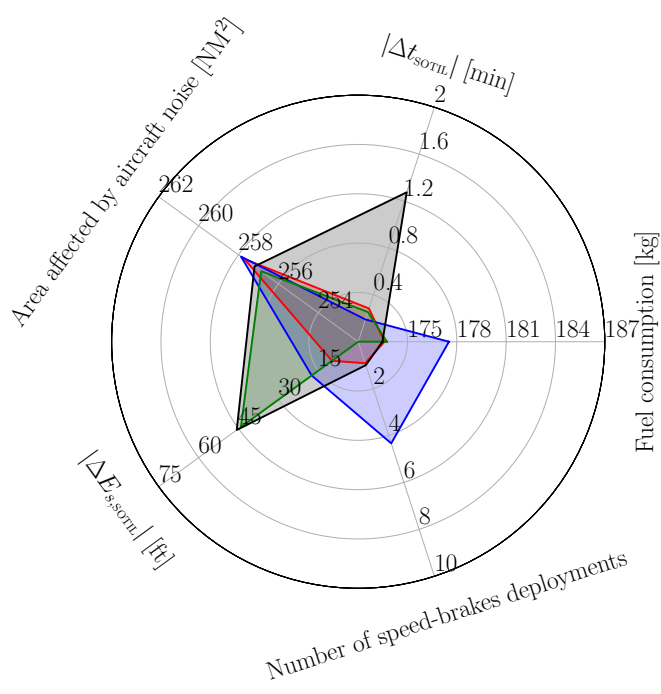

(c) Temperature forecast errors

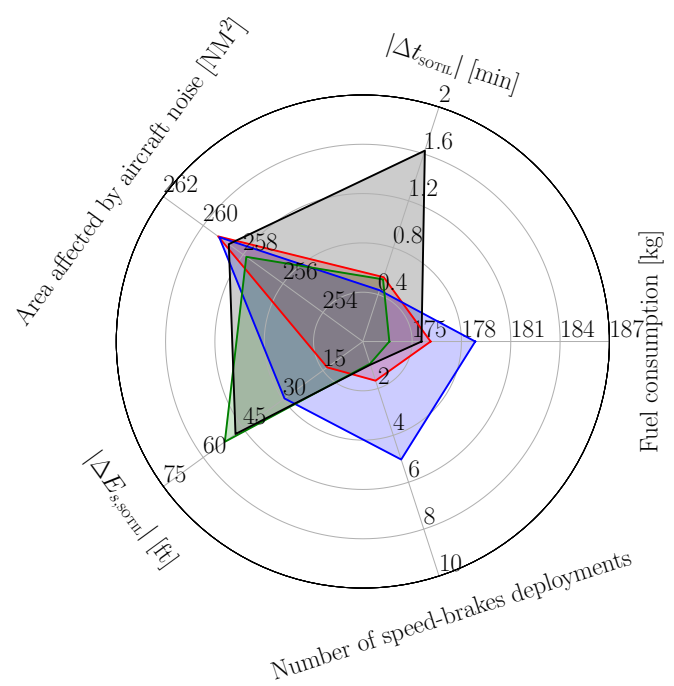

(b) Wind forecast errors

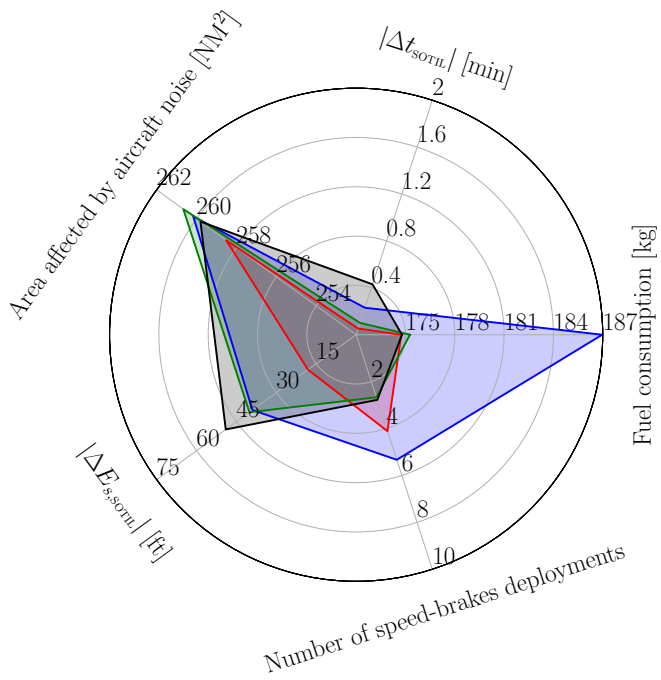

(d) Aircraft performance modelling errors

Fig. 5: Effects of weather forecast and performance modeling errors

\section{B. Comparison of guidance strategies}

The comparison of guidance strategies is illustrated using spider charts, which allow to easily identify the trade-offs of each strategy relative to the others. Figure 5(a) gathers the average results from the 492 simulations; for the remaining subfigures, the set of simulations subject to either wind, temperature or aircraft performance model errors were selected.

According to Fig. 5(a), the average time deviation at SOTIL is lower than two seconds for all the guidance strategies, and less than one second for all those different from the strategic.

This is due to the fact that, in strategic guidance, certain time deviations are allowed before triggering a re-plan. The remaining strategies show better results regarding this metric because their logics continuously correct sustained time deviations, either tactically or by means of continuous re-plans.

Regarding the environmental impact, the tactical strategy shows the worst figures in terms of fuel consumption and noise nuisance, due to the continuous use of throttle and drag devices to correct energy deviations. This strategy also requires the highest number of pilot actions to deploy speed-brakes.

The mechanisms used by the SHMPC are similar to those used by the tactical strategy: energy and time deviations are not monitored, but the elevator, throttle and speed-brakes continuously act to nullify them. However, the corrective actions performed by the SHMPC are mathematically optimal and take into account the remaining trajectory. For this reason, the noise nuisance, fuel consumption and speed-brakes use are comparable with those of the strategic and hybrid strategies.

The hybrid strategy show the best results in terms of environmental impact mitigation and speed-brakes use, proving to exploit the advantages of the strategic and tactical mechanisms.

As expected, the specific energy deviation at SOTIL is higher for the strategic and hybrid strategies, which allow 
deviations up to $100 \mathrm{ft}$ at this fix before triggering a replan. Similarly, the tactical strategy permits specific energy deviations up to $50 \mathrm{ft}$ before applying throttle or deploying speed-brakes. The SHMPC shows the best results in terms of energy deviation, because every 30 seconds the energy deviation is nullified when activating the updated plan.

It should be noted that the fuel consumption shown in Fig. 5 does not consider the approach and landing phases after SOTIL. If this fix were reached with a certain energy deviation, the missing or excessive energy would need to be added by means of additional thrust (fuel consumption) or released by means of speed-brakes (noise nuisance) use, respectively.

Using SHMPC guidance, the aircraft typically reaches the metering fix with small energy deviations, not requiring neither the addition nor subtraction of energy afterwards.

As expected, and according to Fig. 5(b), wind errors have the effect of increasing both absolute time and energy deviations. It is also worth to note that, in aggregated results (and considering the same number of scenarios with head wind than with tail wind), the fuel consumption, noise nuisance and speed-brakes use are similar to those observed in Fig. 5(a).

Figure 5(c) shows that temperature deviations also lead to significant time and energy deviations. On the one hand, all the guidance strategies considered herein execute the CAS plan, leading to TAS errors in presence of temperature deviations. On the other hand, the aircraft performance (and specially the aircraft thrust) strongly depends on the air temperature.

Finally, according to Fig. 5(d), errors in the aircraft performance models are not penalizing the capability to satisfy RTAs, but have a major impact on the environment.

\section{CONCLUSIONS}

This paper compared the performance (in terms of time errors and environmental impact) of four guidance strategy variants of Time and Energy Management Operations (TEMO).

Results from a batch study demonstrated that all the TEMO variants can safely accomplish a Required Time of Arrival (RTA) during a Continuous Descent Operation (CDO) with negligible time deviations, even in presence of weather forecast and aircraft performance modeling errors.

The hybrid strategy shows the best results in terms of environmental impact mitigation, and also requires less pilot actions to deploy speed-brakes. However, due to its strategic behavior with respect to energy deviations, the metering fix is reached with higher energy deviations than for other variants.

The Model Predictive Control (MPC) proved to be the most robust variant regarding energy and time deviations, showing also good environmental impact figures.

A limitation of the experiment presented herein is that the remaining descent from the metering fix to the runway threshold has not been taken into account. If the energy of the aircraft at this fix is too low, additional thrust would be needed after overflying it. Similarly, if the energy is too high, it would be required to use speed-brakes and/or to deploy highlifting devices or the landing gear earlier. In future work, the additional cost caused by these deviations should be estimated.

\section{ACKNOWLEDGMENT}

The authors acknowledge the contributions during the preparation of the experiment of Mr. Jaap Groeneweg and Mr. Michiel Valens from NLR. Much appreciation goes out to $\mathrm{Mr}$. Roel Hogenhuis from NLR for his expertise and advice during the noise computation process.

\section{REFERENCES}

[1] A. Warren and K. Tong, "Development of continuous descent approach concepts for noise abatement," in IEEE/AIAA 21st Digital Avionics Systems Conference (DASC), Irvine, CA, 2002.

[2] J. P. B. Clarke, N. T. Ho, L. Ren, J. A. Brown, K. R. Elmer, K. F. Zou, C. Hunting, D. L. McGregor, B. N. Shivashankara, K. Tong, A. W. Warren, and J. K. Wat, "Continuous descent approach: Design and flight test for Louisville international airport," Journal of Aircraft, vol. 41, no. 5, pp. 1054-1066, 2004.

[3] J. Robinson and M. Kamgarpour, "Benefits of Continuous Descent Operations in High-Density Terminal Airspace Under Scheduling Constraints," in 10th AIAA Aviation Technology, Integration, and Operations (ATIO), Fort Worth, TX, 2010.

[4] J. L. De Prins, F. K. M. Schippers, M. Mulder, M. M. Van Paassen, A. C. In 't Veld, and J.-P. Clarke, "Enhanced Self-Spacing Algorithm for Three-Degree Decelerating Approaches," Journal of Guidance, Control, and Dynamics, vol. 30, no. 2, pp. 576-590, mar 2007.

[5] J. R. Rumbo, M. R. Jackson, and B. E. O'Laughlin, "Aircraft Control System for Reaching a Waypoint at Required Time of Arrival," 2002.

[6] M. R. Jackson, Z. J. Yiyuan, and S. Rhonda A, "Sensitivity of Trajectory Prediction in Air Traf c Management," Journal of Guidance, Control, and Dynamics, vol. 22, no. 2, pp. 219-228, 1999.

[7] D. Garrido-López, L. D’Alto, and R. Gómez Ledesma, "A novel fourdimensional guidance for continuous descent approaches," in AIAA/IEEE 28th Digital Avionics Systems Conference (DASC), 2009.

[8] P. M. A. de Jong, F. J. L. Bussink, R. Verhoeven, N. de Gelder, M. M. V. Paassen, and M. Mulder, "Time and Energy Management during Descent and Approach: a human-in-the-loop study," Journal of Aircraft, vol. 54, no. 1, pp. 177-189, 2017.

[9] P. M. A. de Jong, N. de Gelder, R. Verhoeven, F. J. L. Bussink, R. Kohrs, M. M. van Paassen, and M. Mulder, "Time and Energy Management During Descent and Approach: Batch Simulation Study," Journal of Aircraft, vol. 52, no. 1, pp. 1-14, 2014.

[10] R. Dalmau, R. Verhoeven, N. D. Gelder, and X. Prats, "Performance comparison between TEMO and a typical FMS in presence of CTA and wind uncertainties," in IEEE/AIAA 35th Digital Avionics Systems Conference (DASC), Sacramento, USA, 2016.

[11] M. Diehl, H. G. Bock, J. P. Schlöder, R. Findeisen, Z. Nagy, and F. Allgöwer, "Real-time optimization and nonlinear model predictive control of processes governed by differential-algebraic equations," Journal of Process Control, vol. 12, pp. 577-585, 2002.

[12] A. E. Bryson and Y. Ho, Applied optimal control: optimization, estimation, and control. New York, USA: Taylor and Francis Group, 1975.

[13] J. T. Betts, Practical Methods for Optimal Control and Estimation Using Nonlinear Programming, 2nd ed. SIAM, 2010.

[14] - "Survey of Numerical Methods for Trajectory Optimization," Journal of Guidance, Control, and Dynamics, vol. 21, no. 2, pp. 193207, 1998.

[15] M. R. Almassalkhi, "Optimization and Model-predictive Control for Overload Mitigation in Resilient Power Systems," $\mathrm{PhD}$ thesis, The University of Michigan, 2013.

[16] D. B. Leineweber, H. G. Bock, and J. P. Schlöder, "Fast Direct Methods for Real-Time Optimization of Chemical Processes," in 15th IMACS World Congress on Scientific Computation, Modelling and Applied Mathematics, 1997.

[17] R. W. Pratt, Flight Control Systems: practical issues in design and implementation, ser. Control, R. W. Pratt, Ed. Institution of Engineering and Technology, 2000.

[18] H. Van der Wal, P. Vogel, and F. Wubben, "Voorschrift voor de berekening van de lden en lnight geluidbelasting in db(a) ten gevolge van vliegverkeer van en naar de luchtaven." National Aerospace Laboratorium (NLR), Tech. Rep., July 2001. 